\title{
MASTER
}

The $1 / 2^{+} \rightarrow 1 / 2^{-}$Beta Decay of ${ }^{19} \mathrm{Ne}$ and the Parity

Nonconserving NN Force

E.G. Adelberger, M.M. Hindi, C.D. Hoyle, H.E. Swanson, and R.D. Von Lintig

Physics Department FM-15, University of Washington

Seattle, Washington 98195 USA

\begin{abstract}
We obtain a branching ratio of $(1.20 \pm 0.20) \times 10^{-4}$ for the $\beta^{+}$decay of ${ }^{19} \mathrm{Ne}$ to the $110 \mathrm{keV} 1 / 2^{-}$level of ${ }^{19} \mathrm{~F}$. This transition (presumably dominated by the $\Delta \mathrm{J}^{\pi}=0^{-}$axial charge operator) provides a crucial test of wavefunctions used in interpreting the parity mixing of the ground and $110 \mathrm{keV}$ levels of ${ }^{19}$ F. These wavefunctions, which yield a parity mixing larger than that observed experimentally, also predict too large a $\beta^{+}$decay rate.
\end{abstract}

\section{KEYWORD ABSTRACT}

RADIOACTIVITY ${ }^{19} \mathrm{Ne}$; measured branch to ${ }^{19} \mathrm{~F}(110 \mathrm{keV})$; deduced $\mathrm{ft}$. Compared to she11 model calculation.

PACS classification 23.40.Hc, $21.20 .+\mathrm{n}$

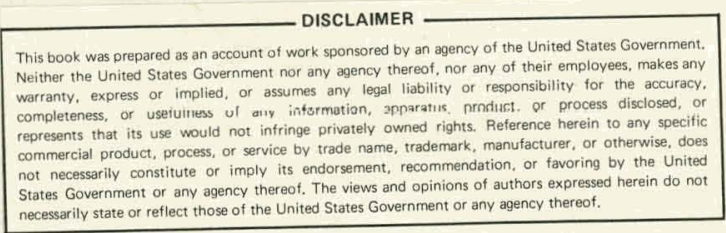




\section{DISCLAIMER}

This report was prepared as an account of work sponsored by an agency of the United States Government. Neither the United States Government nor any agency Thereof, nor any of their employees, makes any warranty, express or implied, or assumes any legal liability or responsibility for the accuracy, completeness, or usefulness of any information, apparatus, product, or process disclosed, or represents that its use would not infringe privately owned rights. Reference herein to any specific commercial product, process, or service by trade name, trademark, manufacturer, or otherwise does not necessarily constitute or imply its endorsement, recommendation, or favoring by the United States Government or any agency thereof. The views and opinions of authors expressed herein do not necessarily state or reflect those of the United States Government or any agency thereof. 


\section{DISCLAIMER}

Portions of this document may be illegible in electronic image products. Images are produced from the best available original document. 
The study of the parity nonconserving (PNC) force between nucleons provides the only practical way to investigate $\Delta S=0$ hadronic weak processes. Recently Desplanques, Donoghue and Holstein ${ }^{(1)}$ have related the PNC emission of mesons by nucleons to the known PNC mesonic decays of the strange baryons. They employed the quark model of hadron structure and the Weinberg-Salam model of the weak interaction to predict the various components of the PNC NN force. Their "best values" are in good quantitative agreement with the measured helicity dependence $(2,3)$ in $p+p$ scattering. However, the $p+p$ results are not sensitive to $\mathrm{PNC} \pi^{ \pm}$exchange (which is pure $\Delta I=1$ ). At present $\pi^{ \pm}$exchange is best probed by the parity mixed doublets in ${ }^{18} \mathrm{~F}(4)$, $19_{\mathrm{F}}(5)$ and ${ }^{21} \mathrm{Ne}^{(6)}$. Haxton, Gibson and Henley ${ }^{(7)}$ and Brown, Richter and Godwin (8) have used large basis shell model wavefunctions to compute the parity mixing in these doublets expected from the "best value" parameters of Desplanques, Donoghue and Holstein. These calculations explain several features of the data, among them the sign of the PNC asymetry in ${ }^{19} \mathrm{~F}$ and the observation of a much larger parity mixing matrix element in ${ }^{19} \mathrm{~F}$ than in ${ }^{21} \mathrm{Ne}$. However the predicted effects in ${ }^{18} \mathrm{~F}$ and ${ }^{19} \mathrm{~F}$ are roughly 3 times larger than the experimental results. Is this due to a deficiency in the nuclear wavefunctions, or because the "best values" of Desplanques, Donoghue and Holstein àre tuo lalge by a factor of n, 3?

There is a way to distinguish between these two possibilities! Consider the first forbidden $\beta$ decays of ${ }^{18} \mathrm{Ne}$ and ${ }^{19} \mathrm{Ne}$ which connect (assuming isospin symmetry) the same levels involved in the parity mixing in ${ }^{18} \mathrm{~F}$ and ${ }^{19} \mathrm{~F}$. These forbidden $\beta$ transitions are dominated by the $\Delta \mathrm{J}^{\pi}=0^{-}$axial charge operator which has a close similarity to the $\Delta J^{\pi}=0^{-}$parity mixing operator. Hence these forlidden $\beta$ decay rates form an excellent test of the wavefunctions used to analyze the parity mixing in ${ }^{18} \mathrm{~F}$ and ${ }^{19} \mathrm{~F}$. 
We recently measured ${ }^{(9)}$ the $\beta$ decay rate of ${ }^{18} \mathrm{Ne}$ to $J^{\pi}=0^{-}, I=0$ level of ${ }^{18} \mathrm{~F}$. The observed rate was roughly 10 times smaller than that predicted by the wavefunction of ref. 7 ! Hence the predicted ${ }^{(7)}$ parity mixing in ${ }^{18} \mathrm{~F}$ should be reduced by $\sim \sqrt{10}$ which removes any discrepancy between the experimental upper limit on the parity mixing and the "best value" PNC $\pi^{ \pm}$exchange coupling given in ref. 1. Haxton ${ }^{(10)}$ has shown that the failure of the wavefunctions was due to neglect of 2 hw excitations on the ${ }^{18} \mathrm{Ne}$ ground state; when these are included he obtains a result in accord with experiment. Could a related phenomenon explain the discrepancy between experiment and theory in the parity mixing in ${ }^{19} \mathrm{~F}$ ? In this Letter we describe a measurement of the $\beta^{+}$decay of ${ }^{19} \mathrm{Ne}$ to the $110 \mathrm{keV} 1 / 2^{-}$level which answers the question in the affirmative.

We produced ${ }^{19} \mathrm{Ne}$ activity by bombarding $\mathrm{SF}_{6}$ gas with $6.4 \mathrm{MeV}$ protons from the University of Washington FN tandem accelerator. A gas transport system (see ref. 9) operating on a $20 \mathrm{sec}$. cycle transferred the irradiated gas to a lucite chamber with $9.5 \mathrm{~mm}$ wall thickness placed in a well-shielded counting station. We observed triple coincidences between $511 \mathrm{keV}$ annihilation quanta detected in two back-to-back $7.6 \mathrm{~cm} \times 7.6 \mathrm{~cm} \mathrm{NaI}$ counters and $\gamma$-rays registered in a $15 \%$ Ge(Li) detector equipped with pileup rejection circuitry. A time-tn-amplitude converter was started on fast 2 -fold.coincidences belween $511 \mathrm{keV}$ events in the $\mathrm{NaI}$ detectors and stopped by fast triggers from the Ge(Li) detector. Scalers recorded the start and stop pulse rates along with the 3 -fold coincidence rate and a rate which gave the pileup losses. From the absence of contaminant lines in the Ge(Li) singles spectra and the observed decay time of activity we conclude that any contaminant activities are negligible for our purposes.

A spectrum of $\gamma$-rays in the Ge(Li) detector in coincidence with two $511 \mathrm{keV}$ quanta is shown in Fig. 1. A smooth bremsstrahlung continuum is 
prominent along with a sharp peak at $110.0 \pm 0.3 \mathrm{keV}$ which contains $531 \pm 88$ counts. We deduced the absolute branching ratio for ${ }^{19}{ }_{\mathrm{Ne}}$ decays to ${ }^{19} \mathrm{~F}(110)$ by dividing the area of the $110 \mathrm{keV}$ peak in the coincidence spectrum by the product of the absolute efficiency of the Ge(Li) detector for $110 \mathrm{keV} \gamma$-rays times the number of 2 -fold coincidences in the NaI detector. An $\sim 30 \%$ correction for pileup losses in the Ge(Li) was incorporated. The absolute point-source efficiency of the Ge(Li) detector and its fast trigger was obtained using calibrated radioactive sources. These sources were placed in the center of the counting cell and singles pulse-height spectra gated by the fast trigger were accumulated and corrected for pileup losses. The measured point source efficiency is shown in Fig. 2. The ${ }^{19} \mathrm{Ne}$ decay measurement involves an extended source. A $-6 \%$ correction to the point source efficiency was inferred from the measured change in the detection efficiency as the radioactive sources were moved around within the counting cell. From these results we obtain a branching ratio of $(1.20 \pm 0.20) \times 10^{-4}$ for the ${ }^{19} \mathrm{Ne} \rightarrow{ }^{19} \mathrm{~F}(1.10)$ decay.

We checked our method by using the same apparatus and procedure to measure the known $\beta^{*}$ branching ratios for ${ }^{18} \mathrm{Ne}+{ }^{18} \mathrm{~F}(1042)$ and ${ }^{22} \mathrm{Na} \rightarrow{ }^{22} \mathrm{Ne}(1275)$. The former activity was made by ${ }^{16} \mathrm{O}\left({ }^{3} \mathrm{He}, \mathrm{n}\right)$ as described in ref. 9, the latter was a commercial radioactive source. Both of our checks required corrections to the 2 -fold coincidence rate which were not needed in the ${ }^{19} \mathrm{Ne}$ case. The ${ }^{22} \mathrm{Na}$ data required a $-13.8 \%$ correction to account for summing of the $1275 \mathrm{keV}$ and $51 \mathrm{lkeV}$ photons in the NaI detectors. The ${ }^{18}$ Ne data required a $-10 \%$ correction to account for the escape from the gas cell of some higher energy $\beta^{+}$'s associated with the ground state branch. The fraction of 2 -fold coincidences due to 18 Ne was inferred by multiscaling the 2 -fold rate. Our measured branching ratios for the $1042 \mathrm{keV} \gamma$-ray in ${ }^{18} \mathrm{Ne}$ decay and the $1275 \mathrm{keV} \gamma-\mathrm{ray}$ in ${ }^{22} \mathrm{Na} \mathrm{B}^{+}$decay are $0.0819 \pm 0.0047$ and $0.956 \pm 0.044$ respectively. These are in reasonable agreement with the accepted 
values of $0.0783 \pm 0.0021^{(11)}$ and 0.999 respectively and give us confidence in our method.

Our branching ratio when combined with the $17.22 \pm 0.02 \mathrm{sec}^{(12)}$ half life of ${ }^{19} \mathrm{Ne}$ corresponds to an ft value of $(1.13 \pm 0.19) \times 10^{-7} \mathrm{sec}$ for the $1 / 2^{+} \rightarrow 1 / 2^{-}$decay. We have computed $f$ assuming an allowed spectrum shape which is reasonable since the dominant axial charge operator is independent of the momentum transfer to the leptons.

Now we turn to some implications of our result. The $1 / 2^{+} \rightarrow 1 / 2^{-} \beta$ decay of ${ }^{19} \mathrm{Ne}$ and the corresponding parity mixing in ${ }^{19} \mathrm{~F}$ differ in some respects from the $\mathrm{O}^{+} \rightarrow \mathrm{O}^{-} \beta$ decays of ${ }^{18} \mathrm{Ne}$ and the analogous parity mixing in ${ }^{18} \mathrm{~F}$ which we have discussed previously ${ }^{(9)}$. The ${ }^{19} \mathrm{Ne}$ decay proceeds by both the vector and axial vector weak currents while the ${ }^{18} \mathrm{Ne}$ decay is purely axial. However it is easy to show that the vector weak current makes a negligible contribution to the ${ }^{19} \mathrm{Ne} \rightarrow{ }^{19} \mathrm{~F}(110)$ decay rate. CVC relates the vector weak current to the isovector electromagnetic current if isospin is a good quantum number for the nuclear states. Thus the vector current contribution to the ${ }^{19} \mathrm{Ne} \rightarrow{ }^{19} \mathrm{~F}(110)$ B decay can be inferred from the El lifetimes ${ }^{(12)}$ of the corresponding $1{ }^{-} 2^{-}$levels in ${ }^{19} \mathrm{~F}$ and ${ }^{19} \mathrm{Ne}$ (see Fig. 3). Using Siegert's theorem to remove trivial $q$ dependence of the matrix elements we obtain

$$
<1 / 2^{-}\left|v^{\beta}\right| 1 / 2^{+}>\propto \frac{1}{2 e}\left(\frac{E_{\hat{B}}}{\hbar c}\right)\left\{\sqrt{\frac{\hbar}{\tau+}\left(\frac{\hbar c}{E_{\gamma}{ }^{+}}\right)^{3}}+\sqrt{\frac{\hbar}{\tau}-\left(\frac{\hbar c}{E_{\gamma}}\right)^{3}}\right\}
$$

where $\tau_{+}$and $\tau_{-}$refer to the lifetimes and $E_{\gamma}{ }^{+}$and $E_{\gamma}{ }^{-}$to the energies of the corresponding El transitions in ${ }^{19} \mathrm{Ne}$ and ${ }^{19} \mathrm{~F}$ respectively while $\mathrm{E}_{B}{ }^{\circ}$ is the energy release in the analogous $\beta^{+}$decay. In computing the $\beta^{+}$decay rate, the rank 1 vector matrix element does not interfere with the dominant rank 0 contribution from the time component of the axial current. Since the El decays are retarded $\left(\sim 10^{-3}\right.$ W.u. $)$ the vector current makes only a small contribution to the measured decay rate and can be neglected. It is reassuring 
'

that the El lifetimes are consistent with the assumed isospin purity of the wavefunctions -- the observed isoscaler E1 matrix element is only $3 \%$ of the observed isovector'matrix element.

A second difference between the mass 18 and mass 19 systems is that the parity mixing in ${ }^{19} \mathrm{~F}$ is a mixture of $\Delta I=0$ and $\Delta I=1$ while that in ${ }^{18} \mathrm{~F}$ is purely $\Delta I=1$. It is only the $\Delta I=1$ component of the parity admixture in ${ }^{19} \mathrm{~F}$ which can be related straightforwardly to the axial $\beta$ decay operator as discussed in refs. 9 and 10 . Hence for ${ }^{19} \mathrm{~F}$ one cannot find a simple expression relating the parity mixing matrix element to the forbidden $\beta$ decay as was possible in ${ }^{18}$. F.

Nevertheless our ${ }^{19} \mathrm{Ne} B$ decay results are a crucial test of wavefunctions used to interpret the parity. mixing in ${ }^{19} \mathrm{~F}$. Our measured decay rate $\omega_{\exp }=(4.8 \pm 0.8) \times 10^{-6} \mathrm{sec}^{-1}$ is roughly 10 times smaller than the rates $\omega_{\text {th }}=4.13 \times 10^{-5} \mathrm{sec}^{-1}$ and $10.4 \times 10^{-5} \mathrm{sec}^{-1}$ predicted (14) using the wavefunctions of refs. 7 and 13 respectively. A similar situation occurred for ${ }^{18} \mathrm{Ne}$ where the measured $\mathrm{O}^{+} \rightarrow \mathrm{O}^{-}$decay rate was approximately 10 times slower than the rate predicted using the wavefunctions of ref. 7 . In that case the discrepancy was removed by incorporating $2 \hbar w$ excitations into the wavefunction of the $\mathrm{O}^{+}$level ${ }^{(10)}$. Unfortunately a similar calculation for ${ }^{19} \mathrm{~F}$ involving $5 \mathrm{p}-3 \mathrm{~h}$ configurations is quite tedious and to our knowledge has not yet been performed. However it is probably fair to assume that whatever shell model deficiencies are responsible for overpredicting the $B$ decay amplitude by roughly a factor of 3 also cause the 3 -fold overprediction of the parity violation in ${ }^{19} \mathrm{~F}$. If so, the "best value" estimates of Desplanques, Donoghue and Holstein would be in remarkable agreement with experiment.

We are grateful to W. C. Haxton for illuminating remarks and for allowing us to quote his unpublished calculations. This work was supported in part by the U.S. Department of Energy. 


\section{REFERENCES}

1. B. Desplanques, J. F. Donoghue and B. R. Holstein, Ann. Phys. 124, 449 (1980)

2. D: Nagle, et al. AIP Conference Proceedings No. 51 (Am. Inst. of Phys., New York, 1978), p. 224.

3. R. Balzer, et al. Phys. Rev. Lett. $\underline{44}, 699$ (1980).

4. C. A. Barnes, et al. Phys. Rev. Lett. 40, 840 (1978).

5. E. G. Adelberger, et al. Phys. Rev. Lett. 34, 402 (1975) and

E. G. Adelberger, Proceedings of Fifth Int. Symp. on Polarization

Phenomena in Nucl. Phys., Santa Fe, NM, 1980, to be published.

6. K. A. Snover, et al. Phys. Rev. Lett. 41,145 (1978).

7. W. C. Haxton, B. F. Gibson, and E. M. Henley, Phys. Rev. Lett. 45,1677 (1980) .

8. B. A. Brown, W. A. Richter and N. S. Godwin, Phys. Rev. Lett. 45, 1681 (1980)

9. E. G. Adelberger, C. D. Hoyle, H. E. Swanson, and R. D. Von Lintig, Phys. Rev. Lett., 46, 695 (1981).

10. W. C. Haxton, Phys. Rev. Lett., 46, 698 (1981).

11. J. C. Hardy, et al. Nucl. Phys. A246, 61 (1975).

12. F. Ajzenberg-Selove, Nucl. Phys. A300, 1 (1978).

13. J. B. McGrory and B. H. Wildenthal, Phys. Rev. C7, 974 (1973).

14. W. C. Haxton, work in progress. 


\section{FIGURE CAPTIONS}

Fig. 1 Spectrum of events in the Ge(Li) counter in coincidence with $511 \mathrm{keV}$ photons detected in two NaI detectors. Random coin- cidences have been subtracted.

Fig. 2 Point source $\gamma$-ray detection efficiency of the Ge(Li) counter. Uncertainties ( $\sim 3 \%$ ) are smaller than the symbols.

Fig. 3 Analogue $\beta$ and $\gamma$ transitions in ${ }^{19} \mathrm{~F}$ and ${ }^{19} \mathrm{Ne}$. 


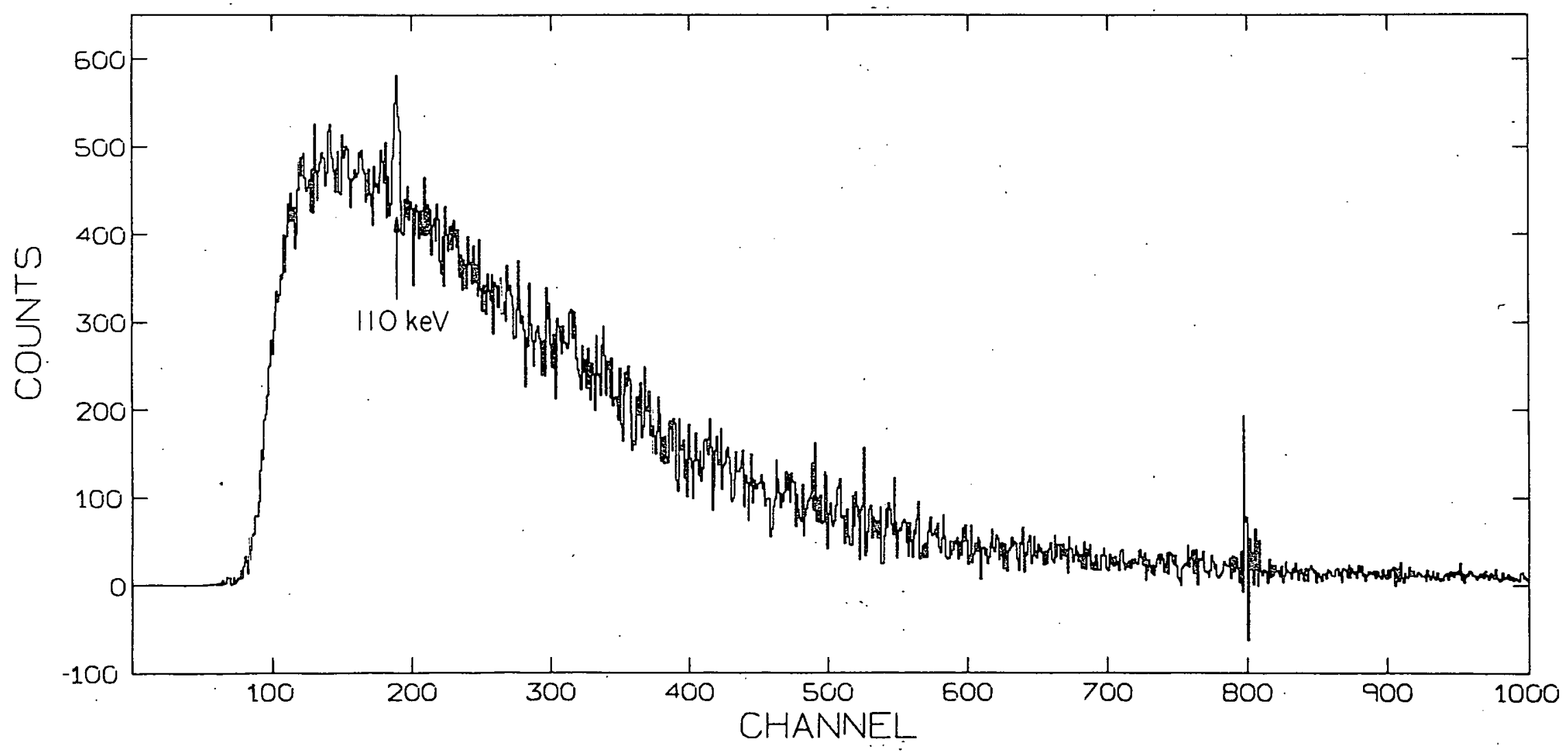




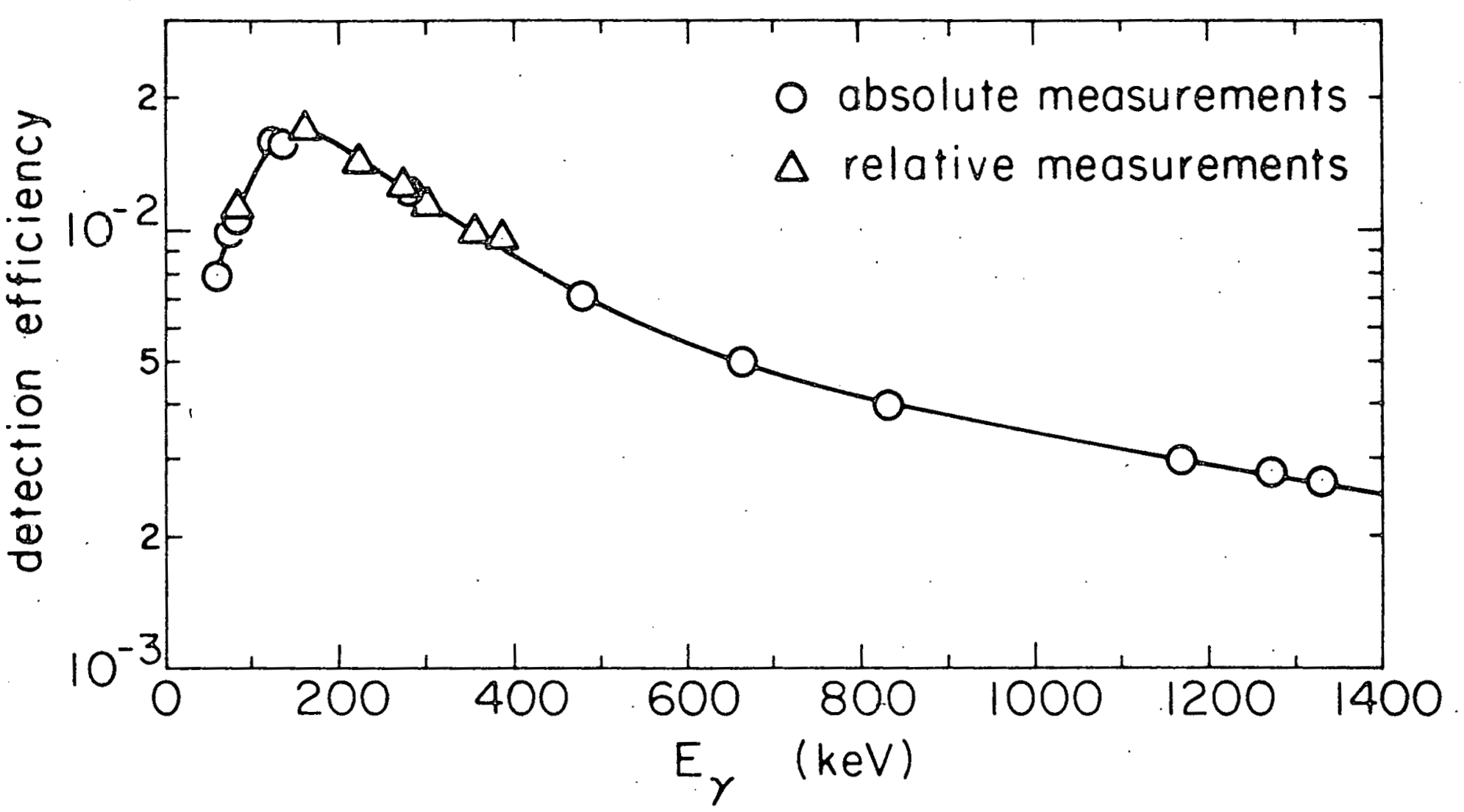




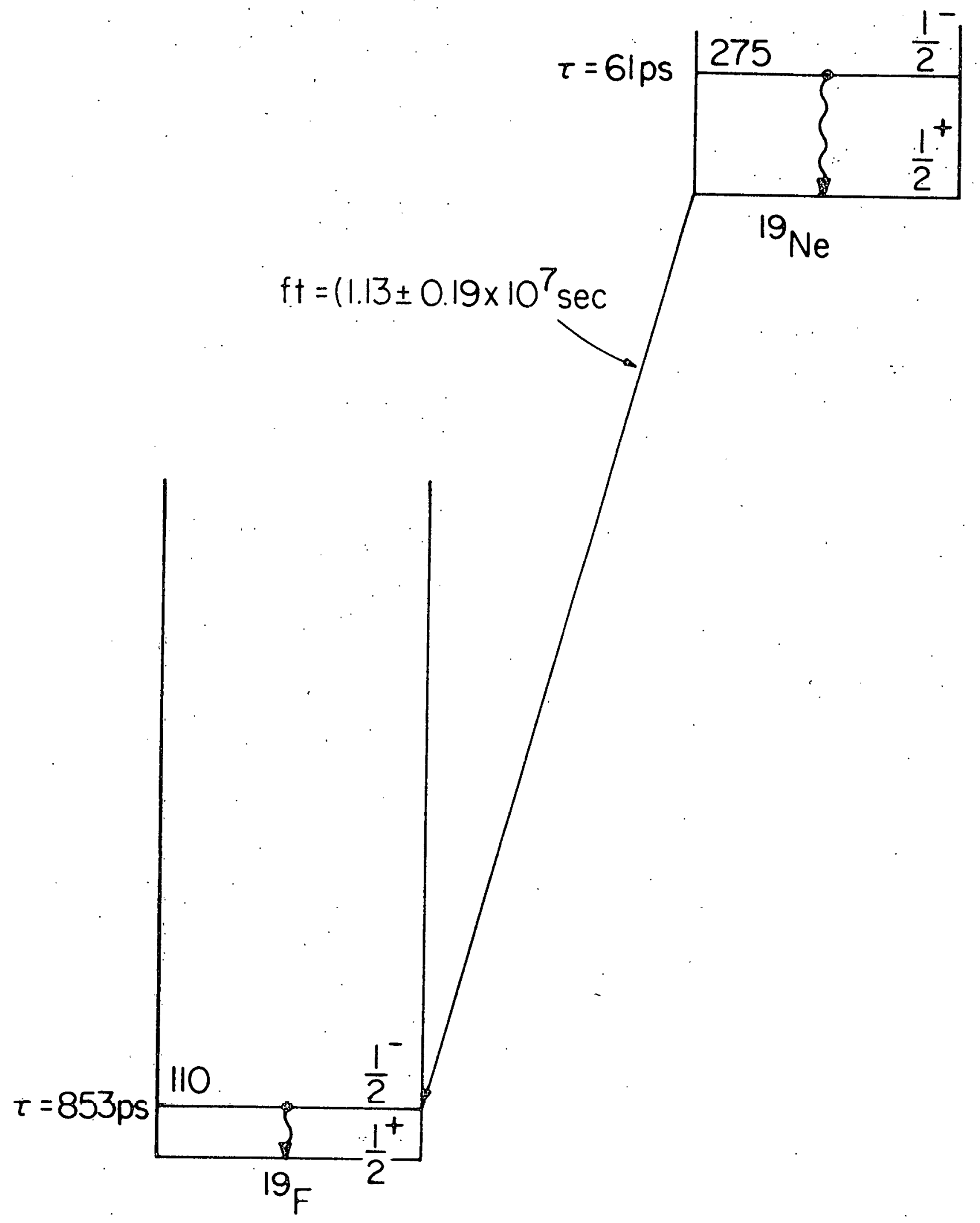

